\title{
Evaluación de factores de riesgo asociados a enfermedad cardiovascular en jóvenes universitarios de la Localidad Santafé en Bogotá, Colombia
}

\author{
Evaluation of risk factors associated with cardiovascular disease in young \\ university students of Santafe Locality from Bogota, Colombia
}

Carmen Cecilia Almonacid Urrego ${ }^{1,2}$, María del Socorro Camarillo Romero ${ }^{3}$, Zulay Gil Murcia ${ }^{1}$, Claudia Yasmin Medina Medina ${ }^{1}$, Jennifer Viviana Rebellón Marulanda ${ }^{1,}$ Hugo Mendieta Zerón ${ }^{2,4}$

\section{Resumen}

Objetivo. Identificar factores de riesgo cardiovascular en población joven de la Localidad Santafé de Bogotá, Colombia. Método. Estudio descriptivo, transversal desarrollado en estudiantes de tres universidades de la Localidad Santafé en Bogotá, con edades comprendidas entre los 16 a 29 años, a quienes se les realizaron exámenes clínicos y paraclínicos y se les aplicó una encuesta de factores de riesgo. Resultados. Se estudiaron 747 estudiantes, encontrando que la prevalencia más alta de factores de riesgo estuvo dada por el consumo de alcohol (96.1\%), que mostró además relación con el aumento de tensión arterial con la prueba Chi cuadrada. Las prevalencias de sedentarismo (63.8\%) y tabaquismo (48.1\%) no presentaron diferencias marcadas en comparación con otros estudios, pero el segundo fue el único que se asoció con el aumento de la tensión arterial sistólica $(P \leq 0.001)$ y diastólica $(P=0.04)$. La prevalencia de obesidad fue similar a la hallada en investigaciones anteriores hechas en Colombia $(2.3 \%)$; de igual manera se encontró asociación entre aquella, la presencia de hipertrigliceridemia $(P=0.001)$ y el incremento en los niveles de glucosa $(P=0.01)$. Los resultados de este estudio indican que los estudiantes universitarios de la Localidad Santafé en Bogotá, tienen como factores de riesgo preponderantes de enfermedad cardiovascular al consumo de alcohol, el tabaquismo y el sedentarismo.

Palabras clave: alcoholismo, Bogotá, factores de riesgo cardiovascular, tabaquismo, hipertensión, universitarios.

\section{Abstract}

Objective. To identify cardiovascular risk factors in young people of Santafé Locality of Bogotá, Colombia. Method. This was a descriptive cross-sectional study conducted at three universities in the Santafe Locality of Bogota, aged 16-29 years, who underwent clinical and laboratory examinations and they were asked to answer a risk factor questionnaire. Results. 747 students were evaluated, finding that the highest prevalence of cardiovascular risk factor was alcohol (96.1\%), also related to an increased blood pressure with the Chi square test. The prevalence of physical inactivity $(63.8 \%)$ and smoking (48.1\%) showed no marked differences compared with other studies, and the second was the only one that was associated with increased systolic $(P \leq 0.001)$ and diastolic blood pressure $(P=$ $0.04)$. The prevalence of obesity was similar to that found in previous research $(2.3 \%)$; and it was also associated to the presence of high levels of triglycerides $(P=0.001)$ and increase in blood glucose $(P$ $=0.01)$. The results of this study indicate that college students in the Santafe Locality of Bogotá, have as main risk factors of cardiovascular disease, alcohol consumption, smoking and physical inactivity.

Keywords: alcoholism, cardiovascular risk factors, Colombia, smoking, hypertension, universities.

${ }^{1}$ Grupo de Investigación ECZA, Universidad Colegio Mayor de Cundinamarca (UCMC), Bogotá, Colombia ${ }^{2}$ Asociación Científica Latina (ASCILA)

${ }^{3}$ Centro de Investigación en Ciencias Médicas, Universidad Autónoma del Estado de México México,

${ }^{3}$ Centro de Investigación en Ciencias Médicas,
${ }^{4}$ Ciprés Grupo Médico (GMC), Toluca, México.

Institución donde se llevó a cabo el trabajo: Universidad Colegio Mayor de Cundinamarca

Correspondencia: calmonacidu@unicolmayor.edu.co

Recibido: 07-02-2016 Aceptado:12-05-2016 


\section{Introducción}

A nivel global cada año fallecen más personas por enfermedades cardiovasculares que por cualquier otra causa, existiendo una clara identificación de los factores de riesgo, modificables y no modificables, que las ocasionan (1).

En la actualidad se acepta que el desarrolloy progreso de las enfermedades crónicas están estrechamente relacionados con factores relacionados con el estilo de vida (2). Este complejo fenómeno se origina por la conjunción de distintos factores que tienen diferente nivel de incidencia (3).

Los factores de riesgo para enfermedades cardiovasculares han sido descritos principalmente en la población adulta, llegando a considerarse como una enfermedad propia del adulto mayor (4). Sin embargo, el patrón epidemiológico ha variado y ya se están informando patrones anormales en todas las edades (5). En este sentido, un estudio realizado en el periodo comprendido entre los ańos 1990 y 2005 informa un aumento del índice de muertes causadas por enfermedades crónicas entre los jóvenes de 15 a 29 años (6).

Dentro de las causas atribuibles a este fenómeno se encuentra el que durante los primeros años de la vida adulta se adquieren ciertos hábitos que incluyen el tipo de dieta, el sedentarismo y el tabaquismo, cuya modificación posterior es difícil, y que minarán la salud cardiovascular del individuo durante ańos posteriores (7).

El panorama de la situación social de Colombia plantea un agravante para la población joven. Aunque en este país la población adolescente y joven presenta altas tasas de mortalidad relacionadas con violencia y suicidio, un porcentaje de dicha población podría estar siendo afectada por enfermedades cardiovasculares al entrar en la vida adulta como consecuencia de largos ańos de exposición a estilos de vida no saludables (4). La tasa de mortalidad en Colombia por enfermedades cardiovasculares en adultos ocupa el primer lugar, siendo el sedentarismo el principal factor de riesgo seguido por la relación colesterol total/HDL > 5, y en tercer lugar por el colesterol total mayor de $200 \mathrm{mg} / \mathrm{dl}(8)$.

Los esquemas actuales de prevención cardiovascular se basan en la detección, el tratamiento y el control de los factores modificables de riesgo para este tipo de enfermedades (9).

Tomando como base todo lo anterior, el presente estudio pretende estimar la frecuencia de factores de riesgo asociados a enfermedad cardiovascular en adultos jóvenes universitarios de tres universidades localizadas en la Localidad Santafé de Bogotá, Colombia.

\section{Materiales y métodos}

Investigación de tipo epidemiológica, de campo, descriptiva, transversal, desarrollada en estudiantes con edades comprendidas entre los 16 y 29 ańos, adscritos a tres universidades ubicadas en la Localidad Santafé de Bogotá, Colombia: Universidad Colegio Mayor de Cundinamarca (UCMC), Universidad Incca de Colombia y Universidad Distrital Francisco José de Caldas.

Como paso previo a la selección de la muestra se realizó una convocatoria invitando a los estudiantes jóvenes de las tres universidades incluidas a vincularse al estudio. Las personas seleccionadas expresaron su consentimiento para participar en el estudio mediante la lectura y firma del formato consentimiento informado.

A todos los participantes se les aplicó una historia clínica, basada en formularios validados. Los formularios utilizados como base para la elaboración de la encuesta fueron: Cuestionario Internacional de Actividad Física (IPAQ) (10), historia dietética para hábitos alimentarios (11), AUDIT para consumo de alcohol (12) y el test de Fagerström para tabaquismo (13). 
Las variables analizadas fueron: edad, género, índice de masa corporal (IMC), obesidad, actividad física, niveles de lípidos (colesterol total, triglicéridos) y lipoproteínas sanguíneas: alta densidad (HDL), baja densidad (LDL) y muy baja densidad (VLDL), niveles séricos de glucemia, presión arterial, tabaquismo y alcoholismo.

A todos los jóvenes se les tomó una muestra de sangre en ayuno de doce horas para determinar el perfil lipídico y los niveles de glucosa sérica y se le midió la tensión arterial con tensiómetro digital. A las personas que presentaron valores de tensión arterial elevados en la primera lectura se les tomaron tres lecturas posteriores, en un día diferente y con un lapso de cinco minutos entre cada una de ellas, dejándose como valor final el de menor valor de hipertensión. El IMC se calculó con la fórmula de Quetelet, empleando la balanza digital Omron para peso. Cada una de las muestras fue centrifugada en el mismo día de su recolección, conservando los sueros a una temperatura de $-80^{\circ} \mathrm{C}$ para su posterior procesamiento.

La cuantificación de los analitos se realizó en el equipo MINDRAY- BS 380 propiedad de la casa comercial ANNAR Diagnóstica, mediante técnicas colorimétricas Spin React. En los pacientes con triglicéridos inferiores a $500 \mathrm{mg} / \mathrm{dl}$ el LDL se calculó mediante la fórmula de Friedewald: [LDL = Colesterol total $-\mathrm{HDL}$ - (triglicéridos/5)]. Con concentraciones de triglicéridos superiores a este valor, el LDL se cuantificó por medición directa.

Para la clasificación de los parámetros a analizar se utilizaron los siguientes consensos internacionales: Presión arterial (PA): de acuerdo con la Sociedad Europea de Hipertensión (ESH) y la Sociedad Europea de Cardiología (ESC) se definió como a) normal (PA sistólica [PAS] de 120 a 129 mmHg y PA diastólica [PAD] de 80 a $84 \mathrm{mmHg}$ ), b) normal-alta (PAS, 130-139 mmHg y PAD, 85-89 $\mathrm{mmHg}$, c) hipertensión grado 1 (PAS, 140-159 mmHg o PAD, 90-99 mmHg), d) hipertensión grado 2 (PAS 160 a 179 mmHg o PAD 100 a 109 $\mathrm{mmHg}$ ) y d) hipertensión grado 3 (PAS $\geq 180$ $\mathrm{mmHg}$ o PAD $\geq 110 \mathrm{mmHg}$ ) (14). Igualmente, se consideró a la clasificación de la JNC 7(15).
Perfil lipídico. De acuerdo con el tercer informe del panel de expertos en detección, evaluación y tratamiento de la hipercolesterolemia en adultos (ATP III) se consideró colesterol normal $<200 \mathrm{mg} /$ $\mathrm{dl}$ y colesterol alto $\geq 200 \mathrm{mg} / \mathrm{dl}$. El colesterol unido a lipoproteínas de alta densidad (cHDL) se consideró bajo $<40 \mathrm{mg} / \mathrm{dl}$ en hombres $\mathrm{y}<50 \mathrm{mg} / \mathrm{dl}$ en mujeres y normal $\geq 41 \mathrm{mg} / \mathrm{dl}$ en hombres $\mathrm{y} \geq 51 \mathrm{mg} / \mathrm{dl}$ en mujeres. Para el colesterol unido a lipoproteínas de baja densidad (cLDL) el valor fue normal $<160 \mathrm{mg} /$ dl y alto $>161 \mathrm{mg} / \mathrm{dl}$. Para los triglicéridos séricos se consideraron los siguientes valores: normal (0-150 $\mathrm{mg} / \mathrm{dl})$ y alto $(>151 \mathrm{mg} / \mathrm{dl})(16)$.

Glucemia. Según la Asociación Latinoamericana de Diabetes (ALAD) se consideraron los siguientes valores: normal $(70-100 \mathrm{mg} / \mathrm{dl})$, alterada (101$125 \mathrm{mg} / \mathrm{dl}$ ) y diabetes ( $\geq 126 \mathrm{mg} / \mathrm{dl})(16)$.

Sobrepeso y obesidad. De acuerdo con la OMS según el IMC se clasificó en: bajo peso $(<18.5)$, normal (entre 18.6 a 24.9), sobrepeso (entre 25 a 29.9) y obesidad ( $\geq 30)$ (1).

Nivel de actividad física. De acuerdo al IPAQ, en versión corta validado para Colombia, se categorizó al nivel de actividad física de la siguiente forma: a) Nivel bajo: sin actividad reportada o no incluido en niveles moderado o alto, b) Nivel Moderado: b.1) 3 o más días de actividad intensa de al menos 20 minutos por día, b.2) 5 o más días de actividad moderada y/o caminata de al menos 30 minutos, b.3) 5 o más días de cualquier combinación de caminata, moderada o intensa, c) Nivel Intenso (o elevado): c.1) Actividad intensa 3 días por semana, c.2) 7 o más días de cualquier combinación (caminata, moderada, intensa) (10).

Ética. El presente proyecto contó con el aval del Comité de Ética de la Universidad Colegio Mayor de Cundinamarca (UCMC) y se rigió bajo los principios éticos y juicios de valor moral de las investigaciones médicas en seres humanos presentados en la declaración de Helsinki, versión 64a Fortaleza-Brasil 2013. Igualmente contempló el Código de Bioética Para el Ejercicio de la Profe- 
sión de Bacteriología y los aspectos éticos especificados en la resolución No. 008430 de 1993, título II, capítulo I (de los aspectos éticos de la investigación en seres humanos) del Ministerio de la Protección Social, Colombia.

Estadística. Se aplicaron las pruebas de normalidad (Kolmogorov-Smirnov y Shapiro-Wilk) para evaluar la distribución de las variables de razón (edad, las relacionadas con el perfil metabólico, la presión arterial y la frecuencia cardiaca). Las variables relacionadas con las funciones fisiológicas y metabólicas y las mediciones antropométricas se transformaron en variables categóricas y se codificaron según los parámetros de normalidad definidos previamente, obteniendo las prevalencias correspondientes. La relación entre las variables de estilos de vida y la presión arterial sistólica y diastólica, y el perfil lipídico con el IMC y la actividad física se establecieron con la prueba Chi cuadrada de diferencia o la prueba de Fisher, este último para los casos con frecuencias esperadas menores de cinco. Las diferencias en las distribuciones del perfil lipídico según sexo, se analizaron con la prueba $U$ de Mann-Whitney. Se consideró significativa una diferencia con un valor $\mathrm{p} \leq 0.05$. El programa estadístico utilizado fue el SPSS versión 17.

\section{Resultados}

Se estudiaron 747 estudiantes pertenecientes a tres universidades ubicadas en la Localidad Santafé en Bogotá, Colombia. 74.6\% (557) de los participantes fueron mujeres y $23.4 \%$ (190) hombres; la mediana de edad fue 20 años, con un rango de 16 a 29 años para hombres y mujeres.

En cuanto a los estilos de vida de la población y como se observa en la Tabla 1, 48.1\% (359) manifestó haber fumado, siendo mayor la frecuencia en los hombres que en las mujeres $(P<0.001)$, mientras que $41.1 \%$ (147) indicó estar fumando en el momento del estudio; 7\% (25) de los fumadores activos consume de once a más de veinte cigarrillos al día. En relación con la ingesta de licor, 96.1\% (718) manifestó haber probado algún tipo de licor, siendo la cerveza y el aguardiente los de mayor consumo con un 69.5\% (499) y $14.1 \%$ (101) respectivamente; $48.8 \%$ (350) expresó consumir alcohol una vez al mes y $1.5 \%$ (11) consume alcohol todos los días. El nivel alto de actividad física de la población es bajo, encontrando que el 63.8\% (477) realiza una baja o nula actividad física y que las mujeres son más inactivas que los hombres $(P=0.001)$.

Tabla 1. Estilo de vida

\begin{tabular}{|c|c|c|c|}
\hline \multicolumn{3}{|c|}{ Variable y frecuencia } & No $(\%)$ \\
\hline \multirow{17}{*}{ Tabaquismo } & \multirow{2}{*}{ Fuma } & SI & $359(48.1)$ \\
\hline & & $\mathrm{NO}$ & $388(51.9)$ \\
\hline & \multirow{4}{*}{ Edad inicio (años) } & 10 a 14 & 89 (24.9) \\
\hline & & $15-19$ & $249(69.6)$ \\
\hline & & $20-24$ & $18(5.0)$ \\
\hline & & $25-29$ & $2(0.6)$ \\
\hline & \multirow{3}{*}{ Actualmente fuma } & SI & $73(20.4)$ \\
\hline & & Ocasionalmente & $74(20.7)$ \\
\hline & & $\mathrm{NO}$ & $210(58.8)$ \\
\hline & \multirow{4}{*}{$\begin{array}{c}\text { Si dejó de fumar, que tiempo tiene } \\
\text { que lo dejó (años) }\end{array}$} & Menos 1 año & $153(72.2)$ \\
\hline & & 1 a 5 & $49(23.1)$ \\
\hline & & 6 a 10 & $9(4.2)$ \\
\hline & & 11 a 15 & $1(0.5)$ \\
\hline & \multirow{4}{*}{ Número de cigarrillos } & 1 a 5 & $307(85.8)$ \\
\hline & & 6 a 10 & $26(7.3)$ \\
\hline & & 11 a 20 & $13(3.6)$ \\
\hline & & $>20$ & $12(3.4)$ \\
\hline
\end{tabular}




\begin{tabular}{|c|c|c|c|}
\hline \multirow{14}{*}{ Alcoholismo } & Toma & $\begin{array}{c}\mathrm{SI} \\
\mathrm{NO}\end{array}$ & $\begin{array}{c}718(96.1) \\
29(3.9)\end{array}$ \\
\hline & \multirow{7}{*}{ Tipo bebida } & Cerveza & $499(69.5)$ \\
\hline & & Aguardiente & $101(14.1)$ \\
\hline & & Vino & $71(9.9)$ \\
\hline & & Ron & $20(2.8)$ \\
\hline & & Whisky & $16(2.2)$ \\
\hline & & Vodka & $7(1.0)$ \\
\hline & & Otro & $4(0.6)$ \\
\hline & \multirow{4}{*}{ Frecuencia de consumo de alcohol } & Una vez al año & $162(22.6)$ \\
\hline & & Una vez al mes & $350(48.8)$ \\
\hline & & Alguna a la semana & $194(27.1)$ \\
\hline & & Todos los días & $11(1.5)$ \\
\hline & \multirow{2}{*}{ Embriaguez } & SI & $379(52.8)$ \\
\hline & & $\mathrm{NO}$ & $339(47.2)$ \\
\hline \multirow{4}{*}{ Actividad física } & \multirow{4}{*}{ Nivel } & No realiza & $80(10.7)$ \\
\hline & & Bajo & $397(53.1)$ \\
\hline & & Medio & $189(25.3)$ \\
\hline & & Alto & $81(10.8)$ \\
\hline
\end{tabular}

Frente a los hábitos alimenticios de la población analizada se encontró que un alto porcentaje de los jóvenes $(87,1 \% ; 650)$ cocina con sal, $38,9 \%$ (290) adiciona nuevamente sal a los alimentos al momento de consumirlos y 78,6\% (586) endulza las bebidas con azúcar. La ingesta de frutas y vegetales demostró que 7,1\% (71) nunca consume frutas, $47,8 \%$ (357) consume vegetales 1 vez por semana y 57,5\% (429) de los jóvenes participantes consumen de un a tres huevos semanalmente. Es de anotar que a pesar de que los hombres no consumen huevos todos los días, cuando lo hacen ingieren un mayor número de los mismos $(\mathrm{p}=0,007)$. Con respecto a la utilización de aceites, 79,6\% (594) de las personas vinculadas al estudio manifestó utilizar el aceite vegetal como principal fuente de grasa para cocinar.
Basándonos en la clasificación de la ESH 7.6\% (57) de todos los estudiantes presentó PAS normalalta al momento del estudio. El porcentaje de participantes con PAS normal-alta e hipertensión arterial sistólica grado uno, fue mayor en los hombres $54.39 \%$ (31), que en las mujeres $4,6 \%$ (26) $(P \leq 0.001)$, sin que hubiese diferencia entre las universidades. De acuerdo a la JNC 7, 2.6\% de la población estudiada estuvo en rango de hipertensión de acuerdo a la PAS (1.7\% con grado 1 y $0.9 \%$ con grado 2 ), mientras que, teniendo en cuenta a la PAD, el porcentaje se elevó a 4.6\% (3.4\% con grado 1 y $1.2 \%$ con grado 2$)$. En cuanto al IMC 15.5\% (116) presentó sobrepeso y 2,2\% (17) obesidad, Tabla 2. Se demostró que las mujeres tienen índices de cintura cadera más altos que los hombres $(P<0.05)$. 
Tabla 2. Parámetros antropométricos

\begin{tabular}{|c|c|c|c|c|c|c|c|c|c|c|}
\hline \multirow{3}{*}{ Parámetro } & & \multicolumn{9}{|c|}{ Universidad } \\
\hline & & \multicolumn{2}{|c|}{ TOTAL } & \multicolumn{2}{|c|}{ UCMC } & \multicolumn{2}{|c|}{ INCCA } & \multicolumn{2}{|c|}{ DISTRITAL } & \multirow[b]{2}{*}{ P } \\
\hline & & No. & $\%$ & No. & $\%$ & No. & $\%$ & No. & $\%$ & \\
\hline \multirow{5}{*}{$\begin{array}{l}\text { Presión sistólica } \\
\text { (percentil 90) }\end{array}$} & Normal & 670 & 89.7 & 331 & 49.4 & 147 & 21.94 & 192 & 28.66 & \\
\hline & Normal alta & 57 & 7.6 & 15 & 26.3 & 34 & 59.65 & 8 & 14.04 & $<0.001$ \\
\hline & HTA grado 1 & 13 & 1.7 & 3 & 23.0 & 6 & 46.15 & 4 & 30.77 & 0.07 \\
\hline & HTA grado 2 & 6 & 0.8 & 0 & 0.00 & 0 & 0.00 & 6 & 100.00 & \\
\hline & HTA grado 3 & 1 & 0.1 & & & & 0.00 & 1 & 100.00 & \\
\hline \multirow{5}{*}{$\begin{array}{l}\text { Presión diastólica } \\
\text { (percentil 90) }\end{array}$} & Normal & 689 & 92.2 & 343 & 49.78 & 152 & 22.06 & 194 & 28.16 & \\
\hline & Normal alta & 24 & 3.2 & 2 & 8.33 & 14 & 58.33 & 8 & 33.33 & $<0.001$ \\
\hline & HTA grado 1 & 25 & 3.3 & 4 & 0.16 & 16 & 64.00 & 5 & 20.00 & $<0.001$ \\
\hline & HTA grado 2 & 4 & 0.5 & 0 & 0.00 & 4 & 100.00 & 0 & 0.00 & \\
\hline & HTA grado 3 & 5 & 0.7 & 0 & 0.00 & 1 & 20.00 & 4 & 80.00 & 0.020 \\
\hline \multirow{2}{*}{ Frecuencia cardiaca } & Normal & 432 & 57.8 & 200 & 46.30 & 119 & 27.55 & 113 & 26.16 & \\
\hline & Alta & 315 & 42.2 & 149 & 47.30 & 68 & 21.59 & 98 & 31.11 & 0.120 \\
\hline \multirow{4}{*}{ IMC } & Normal & 546 & 73.1 & 264 & 48.35 & 134 & 24.54 & 148 & 27.11 & 0.770 \\
\hline & Bajo peso & 68 & 9.1 & 36 & 52.94 & 15 & 22.06 & 17 & 25.00 & \\
\hline & Sobrepeso & 116 & 15.5 & 47 & 40.52 & 30 & 25.86 & 39 & 33.62 & 0.250 \\
\hline & Obesidad & 17 & 2.3 & 2 & 11.76 & 8 & 47.06 & 7 & 41.18 & 0.010 \\
\hline \multirow{3}{*}{$\begin{array}{l}\text { Porcentaje grasa } \\
\text { (hombres) }\end{array}$} & Normal & 106 & 55.8 & 16 & 15.09 & 49 & 46.23 & 41 & 38.68 & \\
\hline & Bajo & 30 & 15.8 & 7 & 23.33 & 9 & 30.00 & 14 & 46.67 & 0.250 \\
\hline & Alto & 54 & 28.4 & 6 & 11.11 & 17 & 31.48 & 31 & 57.41 & 0.070 \\
\hline \multirow{3}{*}{$\begin{array}{l}\text { Porcentaje grasa } \\
\text { (mujeres) }\end{array}$} & Normal & 285 & 51.2 & 160 & 56.14 & 62 & 21.75 & 63 & 22.11 & \\
\hline & Bajo & 64 & 11.5 & 41 & 64.06 & 8 & 12.50 & 15 & 23.44 & 0.240 \\
\hline & Alto & 208 & 37.3 & 119 & 57.21 & 42 & 20.19 & 47 & 22.60 & 0.910 \\
\hline \multirow{2}{*}{$\begin{array}{l}\text { Índice cintura-cadera } \\
\text { (hombres) }\end{array}$} & Normal & 179 & 94.2 & 29 & 16.20 & 72 & 40.22 & 78 & 43.58 & \\
\hline & Alto & 11 & 5.8 & 0 & 0.00 & 3 & 27.27 & 8 & 72.73 & 0.120 \\
\hline \multirow{2}{*}{$\begin{array}{l}\text { Índice cintura-cadera } \\
\text { (mujer) }\end{array}$} & Normal & 133 & 23.9 & 70 & 52.63 & 40 & 30.08 & 23 & 17.29 & \\
\hline & Alto & 424 & 76.1 & 250 & 58.96 & 72 & 16.98 & 102 & 24.06 & 0.030 \\
\hline
\end{tabular}

U de Mann-Whitney

Es de llamar la atención que 1.5\% (11) de los estudiantes tuvo hiperglucemia, $22.8 \%$ (170) presentó hipercolesterolemia y $13.8 \% \quad$ (103) manifestó hipertrigliceridemia. $53.7 \%$ (299) de las mujeres exhibió un bajo nivel de cHDL, mientras que en los hombres el porcentaje fue de
$32.6 \%$ (62); en cuanto al cLDL, $4.1 \%$ (31) de los participantes presentó niveles elevados, Tabla 3. A raíz de nuestro estudio se encontró una asociación estadísticamente significativa de la presencia de PAS normal-alta con el hábito de fumar $(P \leq 0,001)$ y el consumo de alcohol una vez 
a la semana $(P=0.001)$. Sin embargo, la presencia de PAS normal-alta no se relacionó con la actividad física baja. La hipertensión arterial diastólica se presentó con mayor frecuencia entre los hombres $(P=0.001)$, sobresaliendo el hecho del aumento de la hipertensión arterial diastólica grado 1 en los fumadores $68 \%$ (17) con respecto a los no fumadores $(P=0.04)$. En el aspecto metabólico $65.48 \%$ (55) de las mujeres con sobrepeso tuvo niveles bajos de cHDL ( $P=0.029), 11.76 \%$ (2) de los estudiantes obesos presentó hiperglucemia $(P=0.01)$ y hubo asociación estadísticamente significativa entre la obesidad y los altos niveles de triglicéridos $(P=0.001)$.

Tabla 3. Parámetros bioquímicos

\begin{tabular}{|c|c|c|c|c|c|c|}
\hline \multirow{3}{*}{ Parámetro } & & \multicolumn{5}{|c|}{ Universidad } \\
\hline & & TOTAL & UCMC & INCCA & DISTRITAL & \\
\hline & & No. $(\%)$ & No. $(\%)$ & No. $(\%)$ & No. $(\%)$ & $P$ \\
\hline \multirow{3}{*}{ Glucemia } & Normal & $728(97.5)$ & $345(47.39)$ & $177(24.31)$ & $206(28.30)$ & \\
\hline & Baja & $8(1.1)$ & $4(50.00)$ & $0(0.00)$ & $4(50.00)$ & 0.190 \\
\hline & Alta & $11(1.5)$ & $0(0.00)$ & $10(90.91)$ & $1(9.09)$ & $<0.001$ \\
\hline \multirow{2}{*}{ Colesterol total } & Normal & $577(77.2)$ & $272(47.14)$ & $138(23.92)$ & $167(28.94)$ & \\
\hline & Alto & $170(22.8)$ & $77(45.29)$ & $49(28.82)$ & $44(25.88)$ & 0.400 \\
\hline \multirow{2}{*}{ Triglicéridos } & Normal & $644(86.2)$ & $311(48.29)$ & $156(24.22)$ & $177(27.48)$ & \\
\hline & Alto & $103(13.8)$ & $38(36.89)$ & $31(30.10)$ & $34(33.01)$ & 0.09 \\
\hline \multirow{2}{*}{ cHDL (mujeres) } & Normal & $258(46.3)$ & $157(60.85)$ & $70(27.13)$ & $31(12.02)$ & \\
\hline & Bajo & $299(53.7)$ & $163(54.52)$ & $42(14.05)$ & $94(31.44)$ & $\leq 0.001$ \\
\hline \multirow{2}{*}{ cHDL (hombres) } & Normal & $128(67.4)$ & $19(14.84)$ & $68(53.13)$ & $41(32.03)$ & \\
\hline & Bajo & $62(32.6)$ & $10(16.13)$ & $7(11.29)$ & $45(72.58)$ & $\leq 0.001$ \\
\hline \multirow{2}{*}{ cLDL } & Normal & $716(95.9)$ & $335(46.79)$ & $176(24.58)$ & $205(28.63)$ & \\
\hline & Alto & $31(4.1)$ & $14(45.16)$ & $11(35.48)$ & $6(19.35)$ & 0.31 \\
\hline
\end{tabular}

cHDL: colesterol de alta densidad, cLDL: colesterol de baja densidad.

\section{Discusión}

En la población estudiada la mediana de glucemia fue de $87 \mathrm{mg} / \mathrm{dl}$ y $90 \mathrm{mg} / \mathrm{dl}$ para mujeres y hombres respectivamente, coincidiendo con lo publicado en un estudio en población joven realizado por Gallo J. et al., quienes en su estudio tuvieron una media de edad de 24.8 ańos y una media de glucemia de $87.7 \mathrm{mg} / \mathrm{dl}$ en ambos sexos (17), mientras que en México, Camarillo-Romero E. et al. informaron una glucemia de $94.2 \mathrm{mg} / \mathrm{dl}$ en mujeres y $97.2 \mathrm{mg} /$ $\mathrm{dl}$ en hombres de 14 a 16 años de edad (18). Por otra parte, con nuestra metodología, la prevalencia de hiperglucemia fue del $1.5 \%$, hallazgo similar al de otro estudio realizado en adultos jóvenes cartageneros, que reportó un $1 \%$ de prevalencia en la población estudiada (19), siendo bajo en comparación de adolescentes mexicanos con $4.9 \%$ de hiperglucemia (18).

Uno de los factores de riesgo presente en la población estudiada fueron los niveles disminuidos de cHDL, pues $53.7 \%$ de las mujeres y el $32.7 \%$ de los hombres tenían cHDL por debajo del nivel normal. Este comportamiento es similar al observado por Sáez et al. quienes encontraron 
que el $68.4 \%$ de los hombres y $85 \%$ de las mujeres presentaron niveles bajos de cHDL (20) y difieren del reportado por Lozada et al. quienes demostraron una mayor prevalencia en hombres $(68.2 \%)$ que en mujeres (54.5\%) (21).

Aunque estudios realizados en Colombia sobre factores de riesgo asociados a enfermedades cardiovasculares en población universitaria demuestran que entre un $50 \%$ y un $65 \%$ de la población joven tiene concentraciones de cLDL >130 mg/dl (22); en el presente estudio solo el $4.1 \%$ presentó niveles altos de cLDL (>161 mg/dl); estas diferencias se pueden deber a que todos los estudios utilizaron un criterio diferente para la clasificación de sujetos con cLDL alto o a que existen diferencias en cuanto a los estilos de vida de los participantes.

El incremento en colesterol total mostró una frecuencia de $22.8 \%$. Este resultado contrasta con lo encontrado por Alayón (39\%) (19) y Hernández (55\%) (23) en jóvenes universitarios colombianos. Estás discrepancias pueden estar dadas por la diferencia geográfica de los estudios (Bogotá vs Cartagena) y por los diferentes estilos de vida de las dos poblaciones.

Por otra parte, 13.8\% (103) de los jóvenes participantes presentó hipertrigliceridemia, a diferencia de lo encontrado en un estudio realizado en Carabobo, Venezuela, donde el porcentaje fue de 29.54\% (21). Más aún, en Colombia, Hernández et al. dieron a conocer en su estudio que la fracción de lípidos que presentó una mayor prevalencia de niveles alterados fueron los triglicéridos (24.6\%) (23), mientras que en México la prevalencia se ha informado del 29.2\% (18).

Llama la atención que $96.1 \%$ de los participantes ha consumido alcohol, cifra superior al $67.2 \%$ documentado en un estudio de una Universidad de Barranquilla (24). La diferencia se atribuye a que nuestro estudio se realizó en universitarios de todos los semestres, mientras que el de Barranquilla fue en estudiantes de recién ingreso, y como se ha publicado, el consumo de alcohol incrementa conforme se asciende de semestre (25). Si tenemos en cuenta que para el año 2004 en Bogotá 0.7\% de jóvenes consumía licor todos los días y $26 \%$ una a tres veces en el mes (26) y que un estudio posterior del año 2008 documentó que 0.05\% de los adolescentes consumían licor diariamente, $27.2 \%$ mensualmente, y $18.3 \%$ semanalmente (27), vemos que el consumo de alcohol en jóvenes se ha incrementado drásticamente ya que $48.8 \%$ de nuestra población estudiada ingiere alcohol una vez al mes y $1.5 \%$ consume alcohol todos los días.

Frente al tipo de bebida alcohólica que más consumen los jóvenes encuestados, se encontraron la cerveza y el aguardiente como los de mayor consumo con un $69.5 \%$ y $14.1 \%$ respectivamente. Porcentajes que son similares a los encontrados por Cicua D. et al. con $54.7 \%$ y $23.1 \%$ respectivamente (27).

Es de resaltar que en Colombia existe una alta prevalencia de tabaquismo en jóvenes. En un estudio realizado en Bogotá, 41\% de 237 estudiantes universitarios manifestó haber fumado y de ellos $37.6 \%$ eran fumadores habituales (28). De la misma forma, Alonso L. et al reportaron que el $48.7 \%$ de los estudiantes de Barranquilla, Colombia, han fumado alguna vez en su vida y el $35.2 \%$ de los jóvenes son fumadores activos (24). Estos datos concuerdan con los encontrados en el presente estudio donde $48.1 \%$ de los participantes manifestó haber fumado y $41.1 \%$ son fumadores habituales.

Con respecto al promedio de cigarrillos consumidos, un estudio realizado en estudiantes de la Universidad de Manizales mostró que 16.6\% fumaba un promedio de 12.8 cigarrillos semanales (25). Llama la atención que en el presente estudio, del $41.1 \%$ (147) que son fumadores, $7 \%$ consume de 11 a más de 20 cigarrillos al día, incrementando en gran medida la posibilidad de padecer enfermedades cardiovasculares en el futuro, aunque aún falta mucho por investigar en este rubro (29).

En cuanto al IMC, en las universidades estudiadas de la Localidad Santafé en Bogotá, 15.2\% de los participantes presentó sobrepeso y el 2.3\% 
obesidad. Esta misma tendencia se observó en un estudio realizado en 1,865 jóvenes bogotanos donde se halló un sobrepeso de $11.1 \%$ y obesidad de $1.3 \%$ (30). Otros estudios muestran resultados por encima de lo encontrado en nuestra investigación, por ejemplo, Arias et al. en 2007 encontró un sobrepeso del 33.2\% y una obesidad del $16.7 \%$ en 722 niños y adolescentes de Medellín, Colombia (31), dato similar al publicado por Hernández et al. en el año 2010 realizado a 301 jóvenes universitarios cartageneros donde reportó $28.9 \%$ de sobrepeso y $17 \%$ de obesidad (23). Es probable que estas diferencias estén dadas por el contexto de los estudios, por lo que se hace importante realizar estudios incluyendo todas las regiones del país, que aporten datos que permitan establecer el porqué de estas discrepancias.

Páez et al. encontraron en estudiantes de Manizales que el 53\% eran activos, el 39.1\% inactivos y el $15.4 \%$ sedentarios (25). De igual manera, en un estudio de actividad física global en la población adulta de Bogotá con 3,750 individuos se encontraron activos el 36.8\%, moderadamente activos el 26.8\% y sedentarios el 36.4\% (32). Estos hallazgos se relacionan con lo observado en las universidades de la zona centro de Bogotá donde 36.1\% de la población realiza actividad física alta-media, 53.1\% actividad baja y $10.7 \%$ no realiza ningún tipo de actividad física. Esta situación es preocupante ya que el sedentarismo es uno de los comportamientos de riesgo que presenta mayor prevalencia en la actualidad. La práctica de actividad física regular está relacionada con la disminución de los riesgos de enfermedades cardiovasculares y los beneficios son mayores si se inicia en etapas tempranas de la vida (30-33).

Un estudio realizado por Uscátegui et al. mostró que la presión sistólica fue alta para el $1.3 \%$ y la diastólica para el 3.9\% de la población (34); sin embargo, el presente estudio halló PAS normalalta en el $7.6 \%$ de los participantes y $3.3 \%$ de hipertensión arterial diastólica grado 1. Existen pocos estudios en Colombia, que hagan una clasificación con respecto a la tensión arterial sistólica y diastólica; por lo que se hace necesario realizar más estudios que evidencien la relevancia de estos factores en el riesgo cardiovascular.

En esta investigación también se encontró asociación entre hipertrigliceridemia, niveles bajos de cHDL y sobrepeso y obesidad, lo que apoya resultados encontrados por González A et al. quienes determinaron que tanto en hombres como mujeres con obesidad, los niveles de triglicéridos son significativamente más elevados con respecto al grupo sin obesidad (35).

Por otra parte, Graffigna et al. encontraron que los varones presentan mayor PAS y PAD respecto a las mujeres, en un estudio realizado en estudiantes adolescentes de Buenos Aires (36); hallazgos similares se encontraron en este estudio. Los aumentos en la PAS y PAD determinan incrementos en la mortalidad por enfermedad cardiovascular (37). La presión sistólica es un predictor más fuerte de eventos cardiovasculares que la presión diastólica (38).

Sobresale el hecho de que hubo asociación estadísticamente significativa entre el sexo masculino, el hábito de fumar, el consumo de alcohol una vez a la semana y la presencia de PAS normal-alta, en concordancia con Díaz et al. quienes encontraron que un mayor riesgo para desarrollar hipertensión arterial estuvo asociado con las variables sexo, tabaquismo y sobrepeso u obesidad (39).

Finalmente, en cuanto a la presión diastólica, sólo se encontró asociación de ésta con el hábito de fumar. Es importante tener en cuenta que se ha demostrado que el máximo riesgo cardiovascular coexiste cuando las cifras de presión arterial diastólica y sistólica están elevadas, aunque este riesgo no es mayor que cuando sólo existe hipertensión arterial sistólica (40). El consumo de alcohol se asocia a una mayor tasa de mortalidad cardiovascular en bebedores excesivos (40-41). Sin embargo, en el presente estudio se encontró asociación entre el consumo de alcohol una vez por semana y la presencia de tensión arterial diastólica normal-alta. No se encontró asociación entre el 
consumo diario de alcohol y aumento de tensión arterial; lo cual puede estar dado por el tamaño de la muestra y por ende, el bajo porcentaje de consumo diario de alcohol.

Como conclusión, los resultados de este estudio indican que los estudiantes universitarios de la Localidad Santafé en Bogotá, tienen como factores preponderantes de riesgo cardiovascular al consumo de alcohol, el tabaquismo y el sedentarismo.

\section{Agradecimientos}

Agradecimientos: A las bacteriólogas Adriana del Pilar Moreno Valladares y María Vilma Giratá Pedraza por su colaboración en la recolección y procesamiento de las muestras. A las directivas y estudiantes de las universidades participantes, así como a los integrantes del semillero ECZA, por su apoyo incondicional durante todo el desarrollo de la investigación. El presente estudio contó con el apoyo económico de las casas comerciales Annar Diagnóstica Import de Colombia y Becton Dickinson, Colombia. Así mismo, fue cofinanciado por la Universidad Colegio Mayor de Cundinamarca (UCMC), Colombia.

\section{Conflicto de intereses}

Los autores manifiestan no tener ningún conflicto de intereses.

\section{Referencias}

1. Organización Mundial de la Salud. Enfermedades cardiovasculares. Nota informativa: OMS; 2013. Consultado el 13 de abril de 2014. http://www.who.int/mediacentre/factsheets/fs317/es/

2. Cárdenas-Villareal VM1, López Alvarenga JC, Bastarrachea RA, Rizo-Baeza MM, Cortés-Castell E. Prevalencia del síndrome metabólico y sus componentes en adolescentes de la Ciudad de Monterrey, Nuevo León. Arch Cardiol Mex 2010; 80:19-26

3. Rodríguez N, Ríos M, Lozano L, Álvarez M. Percepción de jóvenes universitarios respecto a su salud: conductas y contexto de riesgo. Enseñ e Inv Psicol. 2009; 14:245-60.
4. Sánchez M, Moreno G, Marín M, García L. Factores de riesgo Cardiovascular en poblaciones jóvenes. Rev Sal Pub. 2009; $11: 110-22$.

5. Gotthelfa S, Jubanyb L. Prevalencia de factores de riesgo cardiovascular en adolescentes de escuelas públicas y privadas de la ciudad de Salta, ańo 2009. Rev Ped Arg. 2010; 108:418-26.

6. Cruz Rojo C. Epidemiología de las enfermedades cardiovasculares. Consultado el 13 de abril de 2015. http://personal.us.es/ cruzrojo/cardiovasculares.pdf

7. Laclaustra M, González M, Casasnovas J, Luengo-Fernández E, León-Latre M, Portero-Pérez P, et al. Evolución de los factores de riesgo cardiovascular en jóvenes varones tras 15 años de seguimiento en el estudio Academia General Militar de Zaragoza (AGEMZA). Rev Esp Cardiol. 2006; 59:671-78.

8. Manzur F, Arrieta C. Estudio sociológico y del conocimiento de los factores de riesgo de las enfermedades cardiovasculares en la Costa Caribe Colombiana (Estudio Caribe). Rev Colomb Cardiol. 2005; 12:122-28.

9. Medrano M, Barriusob RP, Boixb R, del Barrio JL, Damián J, Álvarez R, et al. Riesgo coronario atribuible a los factores de riesgo cardiovascular en población española. Rev Esp Cardiol. 2007; 60:1250-6.

10. IPAQ. Cuestionario internacional de actividad física (octubre de 2002). Consultado el 14 de abril de 2014. http://www. ipaq.ki.se/questionnaires/SpanIQSHL7SELFrev230802.pdf

11. INCAP. Manual de instrumentos de evaluación dietética. Guatemala: Serviprensa; 2006. Consultado el 14 de abril de 2015. http://www.incap.int/index.php/es/publicaciones/doc_ view/77-manual-de-instrumentos-de-evaluacion-dietetica

12. Organización Mundial de la Salud. Departamento de Salud Mental y Dependencia de Sustancias. Cuestionario de Identificación de los Trastornos debidos al Consumo de Alcohol (AUDIT). Guatemala: OMS; 2001.

13. Jiménez C, Fagerström K. ¿Hacemos bien el test de Fagerström?. Prev Tab. 2003;5:161-2.

14. Mayoral E, Molina R. Guías del 2003 de la Sociedad Europea de Hipertensión y la Sociedad Europea de Cardiología para el manejo de la hipertensión arterial. J Hypertension. 2003; 21:1011-53.

15. Chobanian AV, Bakris GL, Black HR, Cushman WC, Green LA, Izzo JL Jr, et al. Seventh report of the Joint National Committee on Prevention, Detection, Evaluation, and Treatment of High Blood Pressure. Hypertension. 2003; 42(6):1206-52.

16. Asociación Latinoamericana de Diabetes. Guías ALAD sobre el diagnóstico, control y tratamiento de la diabetes mellitus tipo 2 con medicina basada en la evidencia. Rev ALAD 2013; 1-142.

17. Gallo J, Aristizábal D, Segura A, Correa M, Zapata N. Relación de la resistencia a la insulina con la estructura, la función cardíaca y el metabolismo en adultos jóvenes no obesos. Act Méd Colomb. 2008; 33:117-26.

18. Camarillo-Romero E, Domínguez MV, Amaya-Chávez A, Amaya-Chávez A, Huitrón-Bravo G, Majluf-Cruz A. Dificultades en la clasificación del síndrome metabólico: El ejemplo de los adolescentes en México. Salud Pub Mex. 2010;52:524-27. 
19. Alayón A, Ariza S, Baena K, Lambis L, Martínez L, Benítez L. Búsqueda activa y evaluación de factores de riesgo cardiovascular en adultos jóvenes, Cartagena de Indias, 2007. Biomédica. 2010; 30:238-44.

20. Sáez Y, Bernui I. Prevalencia de factores de riesgo cardiovascular en adolescentes de instituciones educativas. An Fac Med. 2009; 70:259-65.

21. Lozada M, Machado S, Manrique M, Arteaga E, Martínez E, Bastidas G. Factores de riesgo asociado al síndrome metabólico en adolescentes. Gac Méd Caracas. 2008; 116:323-9.

22. Silva S, Rincón M, Dueñas R, Chaves ÁM, Camacho PA, Arenas MA. . El sobrepeso es el factor determinante en la presentación de síndrome coronario agudo en adultos jóvenes colombianos. Rev Colomb Cardiol. 2008; 15:255-62.

23. Hernández J, Herazo Y, Valero M. Frecuencia de factores de riesgo asociados a enfermedades cardiovasculares en población universitaria joven. Rev Salud Pub. 2010; 12:852-64.

24. Alonso L, Pérez M, Alcalá G, Lubo Gálvez A, Consuegra A. Comportamientos de riesgo para la salud en estudiantes colombianos recién ingresados a una universidad privada en Barranquilla (Colombia). Salud Uninorte. 2008; 24:235-47.

25. Páez M, Castaño J. Estilos de vida y salud en estudiantes de la facultad de medicina de la Universidad de Manizales, 2008. Arch Med (Manizales). 2009; 9:146-64.

26. Bonilla R, Torrado MC, Durán E, Quiroga B, Cano G, Sandoval J. Situación actual y prospectiva de la niñez y la juventud en Colombia. Bogotá D.C. 2005; Consultado el 13 de abril de 2015. http://www.emdialogo.uff.br/sites/default/ files/situacao_infancia_e_juv_na_Colombia_0.pdf

27. Cicua D, Méndez M, Muñoz L. Factores en el consumo de alcohol en adolescentes. Pensamiento Psicológico. 2008; 4:115-34.

28. Castaño J, Páez M, Pinzón J, Rojo Bustamante E, Sánchez-Castrillón GA, Torres Ríos JM. Estudio descriptivo sobre tabaquismo en la comunidad estudiantil de la Universidad de Manizales, 2007. Rev Fac Med. 2008; 56:302-17.

29. Pérez M, Pinzón H. Uso del tabaco entre los jóvenes colombianos. Retos para los profesionales en salud pública. Salud Uninorte. 2005; 21:66-75.

30. Vargas M, Becerra F, Prieto E. Evaluación Antropométrica de Estudiantes Universitarios en Bogotá, Colombia. Rev Salud Pub. 2008; 10:433-42.

31. Arias R, Agudelo G. Prevalencia de algunos componentes del síndrome metabólico en escolares y adolescentes con sobrepeso y obesidad. Hallazgos del estudio de factores de riesgo para enfermedad cardiovascular en escolares y adolescentes. Perspect Nut Hum. 2007; 9:11-22.
32. Gómez L, Duperly J, Lucumí D, Gámez R, Venegas AS. Nivel de actividad física global en la población adulta de Bogotá (Colombia). Prevalencia y factores asociados. Gac Sanit. 2004; 19:206-13.

33. Camarillo-Romero E, Domínguez MV, Amaya-Chavez A, Camarillo-Romero Mdel S, Talavera-Piña J, Huitron-Bravo G, et al. Effects of a Physical Activity Program on Markers of Endothelial Dysfunction, Oxidative Stress, and Metabolic Status in Adolescents with Metabolic Syndrome. ISRN Endocrinology. 2012; 2012:970629.

34. Uscátegui RM, Álvarez MC, Laguado I, Soler L. Martínez R. Arias B, et al. Factores de riesgo cardiovascular en nińos de 6 a 18 años de Medellín (Colombia). Ann Ped. 2003; 58:411-7.

35. González A, Amancio O, Islas S, Revilla C, Martín Q, Agustín $\mathrm{E}$, et al. Factores de riesgo cardiovascular asociados a obesidad abdominal en adultos aparentemente sanos. Rev Med IMSS. 2008; 46:273-9.

36. Graffigna M, Honfi M, Soutelo J, Migliano M. Ledesma L, Proietti A et al. Síndrome metabólico y riesgo cardiovascular en estudiantes adolescentes de la ciudad de Buenos Aires. Rev Argent Endocrinol Metab. 2010; 47:14-20.

37. Báez L, Blanco M, Bohórquez R, Botero R, Cuenca G, Márquez $\mathrm{G}$, et al. Guías colombianas para el diagnóstico y tratamiento de la hipertensión arterial. Rev Colomb Cardiol. 2007; 13:187-313.

38. Rosas M, Pastelín G, Martínez J. Hipertensión arterial en México. Guías y recomendaciones para su detección, control y tratamiento. Arch Cardiol Mex. 2004; 74:134-57.

39. Sánchez M, Moreno G, Marín M, García L. Factores de riesgo Cardiovascular en poblaciones jóvenes. Rev Salud Pub. 2009; $11: 110-22$.

40. Secretaria de Salud de México. Enfermedades Cardiovasculares e Hipertensión Arterial. 2001. Consultado el 14 de abril de 2015. http://www.salud.gob.mx/unidades/cdi/documentos/enf_ cardiovasculares.pdf

41. Campuzano, S., et al. (2015). "Determinación de la calidad microbiológica y sanitaria de alimentos preparados vendidos en la vía pública de la ciudad de Bogotá DC." Nova . 